\title{
Post-approval CMC changes with matrix approach
}

Decock Marie, Kaoukab Raji Abdelmoughit and Sennvik Kristina (Global Regulatory Affairs, CMC)

GSK Vaccines, Rue de l'Institut 89, 1330 Rixensart, Belgium

\section{INTRODUCTION}

Importance of post-approval CMC changes for vaccines

- Foster continuous improvement and innovation

- Meet regulatory requirements

Multi-product CMC changes

- Impact on multiple products, e g transfer of manufacturing

(L) facilities, changes in primary containers, raw materials..

- Increased complexity and timings (supply to the patient)

\section{Reduced design strategies}

Ensure a robust evaluation of multi-product changes, applying scientifically-based rationales

- Allow a reliable and timely access to vaccines

- Result in a reduction of the overall number of replicate runs/batches required for evaluation

- Project-dependent and fit-for-purpose

\section{Bracketing}

Process extremes are representative of intermediate conditions (e.g. volume)

\section{Matrixing}

A subset of samples for all factor

combinations is selected for

evaluation (e.g. stability)

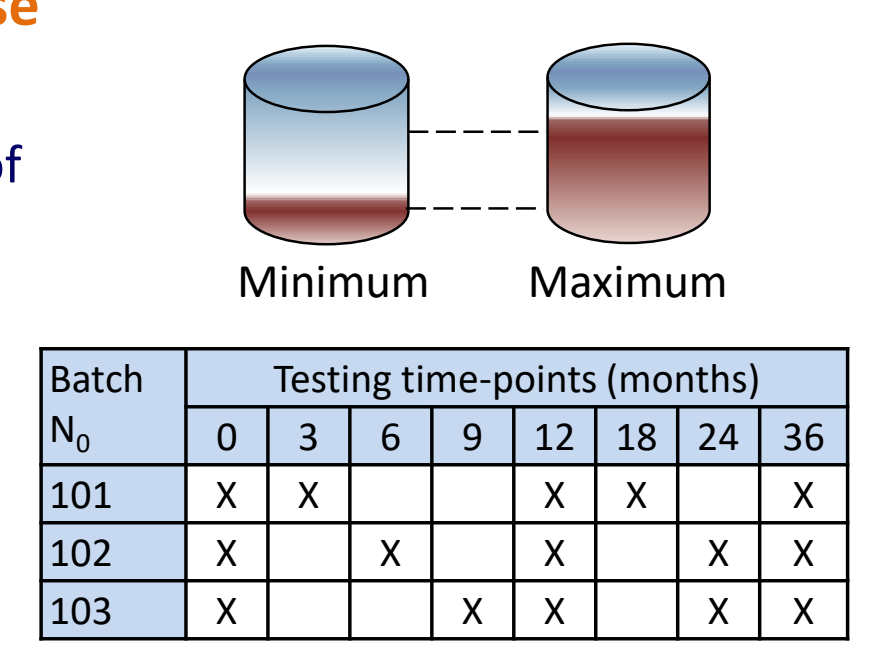

\section{Matrix approach by family grouping}

Grouping of products into families and selecting representatives for validation based on worst-case considerations

Family 1

Family 2

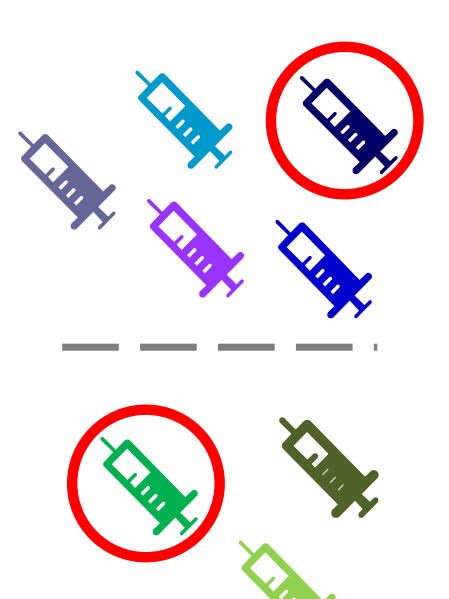

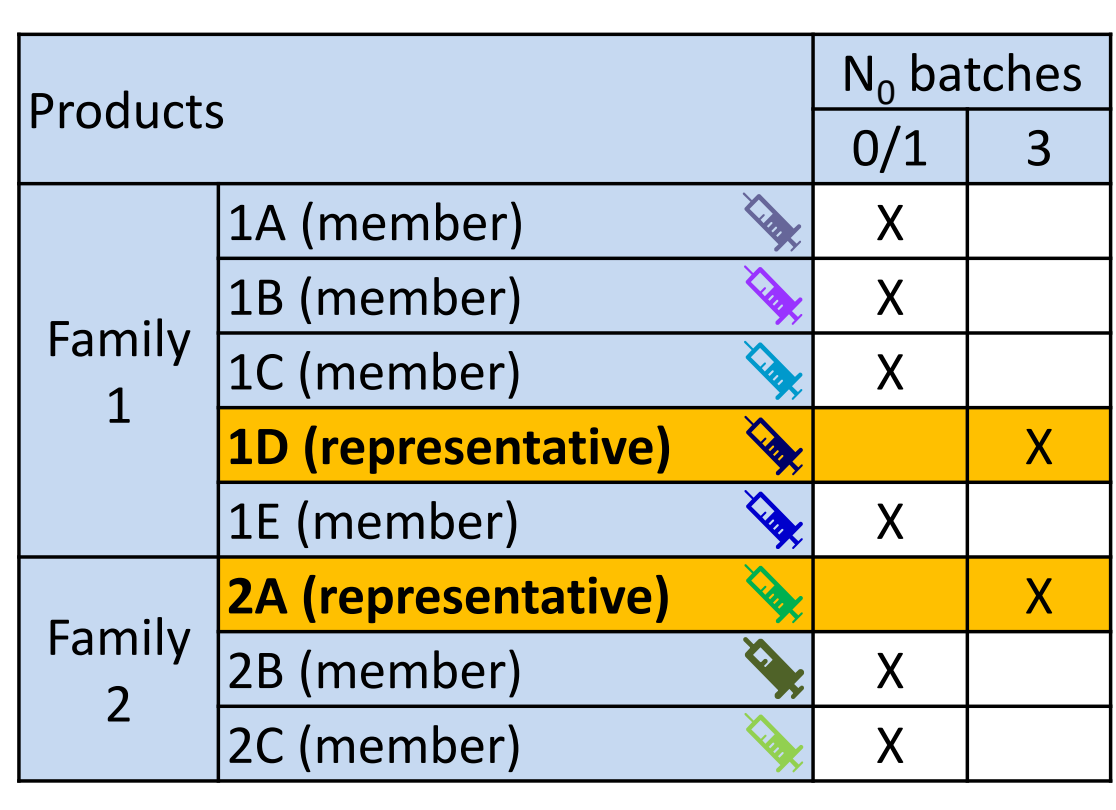

\section{METHOD}

\section{Family grouping and selection of representatives}

- Grouping into families based on manufacturing process, technology, product nature and composition

- Family representative(s) selected based on worst-case considerations (based on process complexity, product composition...)

- Consistency batches are manufactured for the family representative(s)

Additional consistency batch(es) may be manufactured for other family members

- Prior product and process knowledge is high

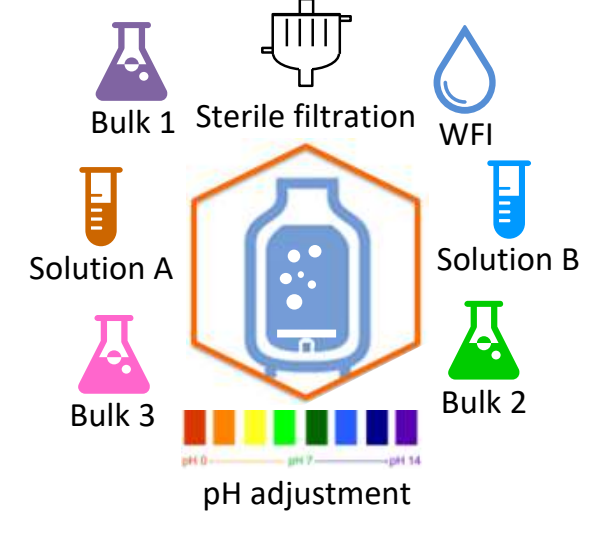

\section{Examples}

- Matrix applied directly to the process under evaluation, e.g. multi-product facility transfer

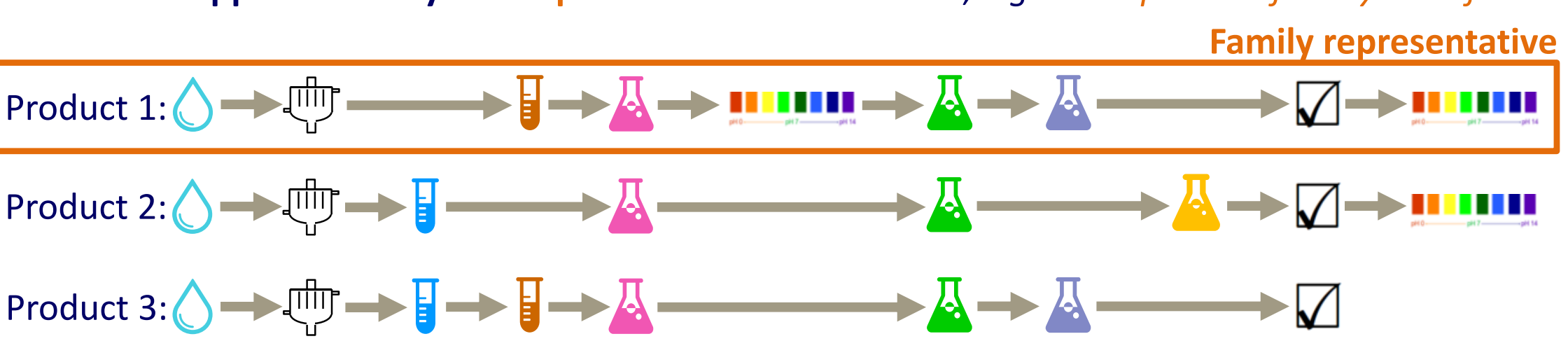

- Matrix applied to generate supportive data to validate a previous manufacturing step, e.g. change in purification equipment of antigen bulk (G)

Bulk (DS): 3 consistency batches with new purification equipment

的 $\rightarrow$ Vaccine (DP): Bulk used in 3 different vaccine formulations 5 ( $n+1$ step)

$$
\text { Product } 1 \text { Product } 2
$$

- Registered products

- Well-established consistency and stability profiles of facilities and equipments Personnel is trained according to internal procedures

WHAT DOES NOT CHANGE

- Manufacturing processes

- Testing (QC, stability) and release

- Operational parameters

e.g. transfer time, holding time
- Classical process qualification

RESULTS $\square$ WHO, EMA, FDA and Health Canada (WHO - "Guidelines for procedures and data requirements for changes to approved

\section{Validation}

Must consider multiple parameters

Must be supported by strong scientific rationales

Approach need to meet internal company requirements

Data obtained for the family representatives are applicable to the whole family grouping vaccines", October 2014)

Regulatory

Family representative(s) may not be registered in every country Additional data may need to be provided (commercial lots)

- Single-source countries, manufacturing routes

- Changes in the regulatory environment

CONCLUSIONS - The matrix is an alternate risk-based streamlined approach to reduce the number of consistency batches applying scientifically-based rationales

- The matrix approach allows robust validation together with optimized project timelines

- Progress still needed to fully embed the concept in regulatory guidelines

This work was sponsored by GlaxoSmithKline Biologicals SA. All authors have declared the following interests: Decock Marie, Kaoukab-Raji Abdelmoughit and Sennvik Kristina are employees of the GSK group of companies. 